\title{
Lymphocyte reduction induced by hindlimb unloading: distinct mechanisms in the spleen and thymus
}

\author{
Li Xin WEI, Jian Nian ZHOU, ARthur I ROBERTS, Yu Fang SHI* \\ Department of Molecular Genetics, Microbiology and Immunology, University of Medicine and Dentistry of \\ New Jersey-Robert Wood Johnson Medical School, 661 Hoes Lane, Piscataway, New Jersey 08854, USA \\ E-mail: yufang.shi@umdnj.edu
}

\begin{abstract}
Hindlimb unloading (HU) in rodent is a well-accepted ground-based model used to simulate some of the conditions of space flight and reproduce its deleterious effects on the musculoskeletal, cardiovascular and immune systems. In this study, the effects of HU on lymphocyte homeostasis in the spleen and thymus of mice were examined. HU was found to drastically deplete various cell populations in the spleen and thymus. These changes are likely to be mediated by apoptosis, since DNA strand breaks indicative of apoptosis were detected by terminal deoxynucleotidyl transferase-mediated nick end-labeling in both splenocytes and thymocytes. Surprisingly, administration of opioid antagonists or interference with the Fas-FasL interaction was able to block HU-induced reductions of splenocytes, but not thymocytes. On the other hand, steroid receptor antagonists blocked the reduction of lymphocyte numbers in both spleen and thymus. Therefore, the effects of HU on the homeostasis of splenocytes and thymocytes must be exerted through distinct mechanisms.
\end{abstract}

Key words: hindlimb unloading, apoptosis, stress, lymphocytes.

\section{INTRODUCTION}

Bi-directional interactions between the immune and neuroendocrine systems play a critical role in the maintenance of immune homeostasis[1,2]. These interactions influence antibody and cytokine responses, cytolytic ativity and proliferation of lymphocytes[3], as well as the lymphocyte pool in various organs $[4,5]$. Cytokines produced during immune responses also exert effects on hypothalamic-pituitary-adrenal (HPA) hormone secretion[6] and neural signal transmission[7, 8]. In addition, cells of the immune system produce some hormones and neurotransmitters and express receptors for them. Recent progress in psychoneuroimmunology has revealed that stress can affect the immune system through these interactions[9-11]. Numerous studies have shown that exhausting physical activity and severe environmental and/or psychological stress have strong suppressive effects on the immune system[12]. Such suppression has signifi-

\footnotetext{
"Corresponding author. Dr. Yufang SHI, Tel: 732-235-4501; Fax: 732-235-4505, E-mail: yufang.shi@umdnj.edu Received Aug-20-2002 Revised Sep-17-2003 Accepted Oct-28-2003
}

cant implications for the susceptibility onset, course, and outcome of diseases such as cancer [13-15], autoimmunity $[15,16]$ and infections $[15,17]$.

Using various model systems, it has been demonstrated that stress may either enhance or impair immune functions, depending on its mode and duration[18, 19]. It is generally accepted that acute stress boosts immune system function, while chronic stress is often suppressive. The immuosuppression resulting from chronic stress is due in part to a reduction in lymphocyte numbers[20-22]. Consequently, stress-induced lymphopenia has been observed in surgical patients[23], over-exercised athletes [24], persons under various psychological stresses[25, $26]$, and animals subjected to physical restraint $[15,27]$. While several immune effects have been shown to be associated with stress, the exact mechanisms remain to be elucidated, especially in different organs of the immune system. Using a wide variety of techniques to detect apoptosis, others and we have found that lymphopenia observed after exposure to stress is in fact due to the induction of apoptosis. 
Apoptosis is an evolutionarily conserved 'cell suicide' program present in all nucleated metazoan cells[28-30]. Despite its highly conserved nature, it is only recently that some of the molecular mechanisms underlying apoptosis have been identified[31]. Apoptosis is now known to be an active cell death process characterized by activation of proteases, auto-destruction of chromatin, nuclear condensation, and cellular membrane blebbing [32]. This gene-directed process is responsible for the widespread and deliberate elimination of excessive cells during development and in pathophysiological processes. In the immune system, apoptosis occurs physiologically during lymphocyte repertoire selection and in the course of normal immune responses. One of the better-characterized systems in which apoptosis occurs is the activationinduced cell death (AICD) of T cells, in which apoptosis can be induced specifically in immature thymocytes in vivo by administration of antibodies against the TCR complex[33,34]. This was also observed in fetal thymic organ culture, using either antibodies against CD3[35] or the superantigen, staphylococcal enterotoxin $\mathrm{B}[36]$. AICD in immature thymocytes is believed to drive the negative selection of autoreactive T-cells during their development in the thymus. Mature T cells are generally believed to be resistant to AICD, but when primary activated mature $T$ cells are activated again, the majority do undergo apoptosis, a finding confirmed in both mouse [37] and human cells[38]. AICD of mature T cells is considered a fundamental mechanism for the elimination of excess peripheral lymphocytes, which is essential for maintaining immune homeostasis following an immune response. Defects in this programmed removal mechanism could therefore lead to an accumulation of potentially autoreactive lymphocytes. On the other hand, excessive apoptosis may cause immunodeficiency, a prime example being HIV-induced helper T cell apoptosis in AIDS.

Fas antigen is a type I membrane protein of $45 \mathrm{kDa}$ that belongs to the tumor necrosis factor (TNF) receptor/ nerve growth factor receptor family. It is expressed on a variety of cell types including activated T cells and $\mathrm{B}$ cells, hepatocytes, and ovarian epithelial cells[39]. It is also detected in solid tumors of the breast, ovary, colon, prostate, and liver. Ligation of this molecule with specific antibodies or its natural ligand (FasL) usually induces rapid apoptotic cell death. FasL is a $40 \mathrm{kDa}$ type II membrane protein belonging to the TNF family[40, 41]. Its expression has been detected in activated $\mathrm{T}$ cells,
NK cells, Sertoli cells of the testis, corneal epithelium, and the retina of the eye. Mutation of either Fas (lpr) or FasL (gld) results in lymphadenopathy and splenomegaly, and accelerates autoimmune disease in both mice and humans[42]. Our studies have revealed that the expression of Fas can be strongly induced by opioids, whether exogenously administered or produced endogenously[43, 44].

During space flight, immune system function is dramatically affected. A number of laboratories have demonstrated that space flight has profound effects on many immunological parameters in humans and animals, including decreases in leukocyte blastogenesis and natural killer cell activity, alterations in leukocyte subset distribution and cytokine production, abnormal macrophage maturation and activity, and increased monocytosis and lymphopenia[45]. These effects may result from stressors encountered during space flight.

Hindlimb unloading (HU) in rodents is a well-accepted ground-based model system used to simulate some of the deleterious conditions of space flight. In this study, we show that splenocyte and thymocyte numbers are dramatically reduced in mice subjected to $\mathrm{HU}$ and that blocking of opioid receptors or the Fas-FasL interaction nearly eliminates the reduction of splenocytes, but not thymocytes, suggesting that the mechanism mediating $\mathrm{HU}$-induced apoptosis in peripheral lymphoid organs differs from that in the thymus.

\section{MATERIALS AND METHODS}

\section{Animals}

Six to eight-week-old male Balb/c mice were purchased from the National Cancer Institute (Fredrick, MD). They were housed in barrier conditions under controlled temperature and light cycle in the vivarium of Robert Wood Johnson Medical School, with food and water provided ad libitum. Animals were allowed to rest for at least one week following shipment and were age-matched in each experiment. Experimental procedures were approved by the Institutional Animal Care and Use Committee and carried out under ve-terinary supervision.

\section{Hindlimb unloading}

Mice were individually housed in cages manufactured according to Chapes, et al. [46]. Food and water were provided ad libitum, and the amounts consumed were similar in both HU-treated and control mice. HU-treated mice, however, lost about $10 \%$ of their body weight during the course of the treatment. HU was carried out as described by Belay, et al.[47]; in this system, mice are suspended by the tail using a strip of adhesive surgical tape that is attached to a chain hanging from a pulley, such that only the forelimbs touch the floor. The pulley suspension allows freedom of movement laterally while maintaining a $25-30^{\circ}$ angle between the longitudinal body axis and 
cage floor, preventing load bearing on the hind limbs. Control mice were housed under similar conditions, except that, while the tail was taped, it was not raised, and all limbs were in contact with the floor. In some experiments, specific receptor-ligand interactions were blocked by i.p. injection of mice $2 \mathrm{~h}$. before HU-treatment with one of several reagents (dissolved in $100 \mu \mathrm{l}$ physiological saline). These were: Fas-Fc fusion protein, a soluble chimera of the $C \gamma 1$ region of human Ig and the extracellular domain of murine Fas that binds FasL [48], or anti-FasL antibody (MFL3, BD-Pharmingen), each at 150 ug, to neutralize FasL; naltrexone, an opioid-receptor blocker, or RU486, a corticosteroid receptor antagonist, each at $10 \mathrm{mg} / \mathrm{kg}$ (Sigma Chemical, St. Louis, MO). All experiments were repeated at least twice.

\section{Cell counts and surface marker analysis}

Single-cell suspensions were prepared from spleen and thymus by grinding minced tissue from the entire organ between the frosted ends of two glass microscope slides. Cells were then washed, resuspended in complete medium, and enumerated on a hemacytometer. To determine the expression of specific surface markers, cells were stained with FITC- or PE-conjugated antibodies against CD4, CD8, or CD19 according to standard methods, and analyzed on a FACScan flow cytometer by two-color analysis (BD Bioscences, Mountain View, CA).

\section{TUNEL assay}

To detect apoptotic cells, terminal deoxynucleotidyl transferasemediated deoxyuridine nick end-labeling (TUNEL) staining was performed on lymphoid organs in situ using a commercially- available kit from Intergen (Purchase, NY). Accordingly, spleen and thymus samples were fixed with $10 \%$ formalin, embedded in paraffin, and thin sections stained by TUNEL according to kit instructions. Thus, 3'- OH ends of fragmented nucleosomal DNA of apoptotic cells were specifically labeled using exogenous terminal transferase and dUTP, then detected with fluorescein-labeled antibody specific for deoxyuridine. Sections were then examined by fluorescence microscopy for positive staining.

\section{Statistical analysis}

Each experimental group consisted of at least four mice and all experiments were done at least twice. Differences in subpopulation of lymphocytes between treatment and control group were analyzed by Student' $s$ t-test, and differences between treatment groups were analyzed by ANOVA.

\section{RESULTS}

Hindlimb unloading (HU) causes severe depletion of lymphocytes in the spleen and thymus

We have reported previously that mice subjected to confinement stress show significant reductions in splenocyte numbers[44]. We have now observed a similar loss of cellularity in mice after hindlimb unloading (HU), a ground-based animal model simulating some conditions of space flight. In these experiments, mice were suspended by tail traction (body axis at $25-30^{\circ}$ angle with cage floor) so that only the forelegs contacted the floor. Controls were housed under identical conditions, with the tail taped, but not suspended. Mice from each group were sacrificed after the indicated intervals, and cell number and phenotype in the spleen and thymus determined. As shown in Table 1, after two days of HU treatment, the number of $\mathrm{T}$ cells in the spleen were reduced by $45 \%$, while $\mathrm{CD} 19^{+}$ B cells were slightly less affected. After 4 days, $50 \%$ of splenic $\mathrm{T}$ cells and $\mathrm{B}$ cells were lost. In the thymus, immature $\mathrm{CD} 4^{+} \mathrm{CD} 8^{+}$thymocytes were the most sensitive, dropping by $70 \%$ after only two days. The total number of mature single positive $\left(\mathrm{CD} 4^{+} \mathrm{CD} 8^{-}\right.$or $\mathrm{CD} 4-\mathrm{CD} 8^{+}$) thymocytes was also reduced, but to a lesser degree, each decreasing by more than $60 \%$ on day 7 . Thus, HU caused dramatic drops in thymic and splenic lymphocyte numbers.

\section{Tab 1. Effect of Hindlimb Unloading on Splenocytes and Thymocytes*}

\begin{tabular}{|c|c|c|c|c|c|c|c|c|}
\hline & \multicolumn{4}{|c|}{ Spleen (Davs Unloaded) } & \multicolumn{4}{|c|}{ Thymus (Davs Unloaded) } \\
\hline & Control & $2 d$ & $4 d$ & $10 \mathrm{~d}$ & Control & $2 d$ & $7 d$ & 10d \\
\hline No of Mice & 8 & 5 & 5 & 10 & 8 & 5 & 5 & 6 \\
\hline $\mathrm{CD}^{+} \mathrm{CD8}^{+}$ & - & - & -- & -- & $93.9 \pm 12.6$ & $29.5 \pm 18.4$ & $24.8 \pm 7.5$ & $22.0 \pm 5.8$ \\
\hline $\mathrm{CD}_{4}^{+} \mathrm{CD8}^{-}$ & $25.4 \pm 4.4$ & $15.1 \pm 3.1$ & $11.5 \pm 3.6$ & $10.1 \pm 1.3$ & $14.8 \pm 2.9$ & $12.6 \pm 3.6 \#$ & $5.8 \pm 1.4$ & $3.2 \pm 0.5$ \\
\hline $\mathrm{CD}^{-\mathrm{CD}^{+}}{ }^{+}$ & $14.8 \pm 2.7$ & $7.8 \pm 1.8$ & $7.6 \pm 2.3$ & $7.6 \pm 1.4$ & $5.4 \pm 1.8$ & $4.1 \pm 1.0 \#$ & $1.9 \pm 0.8$ & $0.9 \pm 0.2$ \\
\hline $\mathrm{CD}^{+}{ }^{+}$ & $52.7 \pm 6.7$ & $41.9 \pm 4.9$ & $27.3 \pm 7.0$ & $19.9 \pm 5.3$ & -- & -- & -- & -- \\
\hline
\end{tabular}

*Male Balb/c mice at 8 weeks old were subjected to hindlimb unloading for different times and cell subpopulations were analyzed by flow cytometry. The values represent total number of cells of each phenotype (mean $\left.\pm \mathrm{SD}, \mathrm{x} 10^{6}\right)$. Student's t test revealed significant reductions at all time points as compared to control, $\mathrm{p}<0.01$, except marked by \#. 
Hindlimb unloading induces apoptosis in the spleen and thymus

HU-induced splenocyte and thymocyte losses may be mediated by two possible mechanisms: cell emigration or cell death by apoptosis. Therefore, we examined the presence of apoptotic cells in histological sections of spleen and thymus using the TUNEL staining technique to reveal DNA strand breaks typical of apoptosis. We found that a significant number of cells in the spleen underwent apoptosis after mice were subjected to $\mathrm{HU}$, while only a few apoptotic cells were observed in tissue sections of control mice (Fig 1). Thus, HU-initiated depletions in splenocyte and thymocyte numbers were due, at least in part, to the induction of apoptosis.
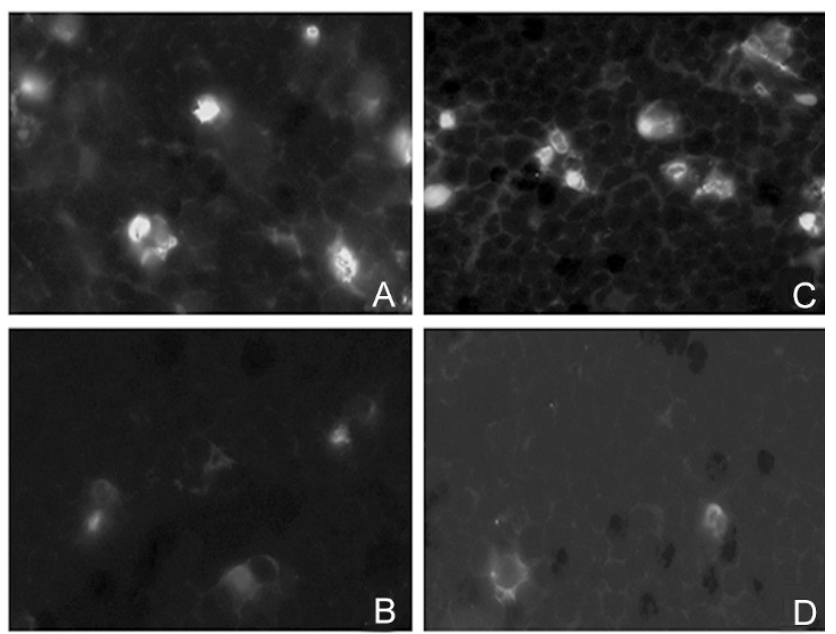

Fig 1. Apoptosis in thymus and spleen after HU-treatment as detected by TUNEL staining. Tissue sections of organs from mice subjected to HU for 2 days were stained by the TUNEL method to reveal DNA strand breaks indicative of apoptosis and then examined by fluorescence microscopy. Both spleen $(\mathbf{A}, \mathbf{B})$ and thymus $(\mathbf{C}, \mathbf{D})$ from HU-treated mice $(\mathbf{A}, \mathbf{C})$ show a high frequency of apoptotic cells compared to controls $(\mathbf{B}, \mathbf{D})$ as indicated by positive fluorescent staining in these representative micrographs (400X).

\section{The role of endogenous opioids and corticosteroids}

Our previous investigations revealed that splenocyte reductions resulting from confinement stress depend on endogenous opioids, since blocking of opioid receptors with naltrexone or naloxone abolished this effect[44]. This finding was recently corroborated using $\mu$-opioid receptor knockout mice[49]. To determine whether endogenous opioids play a similar role in the lymphocyte reduction seen in HU treatment, Balb/c mice were injected with naltrexone $(10 \mathrm{mg} / \mathrm{kg}$ i.p.) $2 \mathrm{~h}$ before, and daily thereafter, while subjected to HU for 2 days. We found that naltrexone dramatically inhibited splenocyte depletion (Fig 2A). Daily administration of RU486, a steroid receptor antagonist, similarly prevented splenocyte depletion, an interesting finding considering that some effects of endogenous opioids are exerted through the induction of corticosteroid production[50,51]. It is also possible that endogenous opioids and corticosteriods each act independently on splenocytes. In any case, the link between corticosteriods and endogenous opioids in the modulation of splenocyte homeostasis warrants further investigation. In contrast to the spleen, thymocyte reduction after HU was blocked by RU486, but not naltrexone (Fig 2B), indicating a critical role only for corticosteriods in this process. This is in agreement with the reported key role of corticosteriods in opioid-mediated thymocyte reduction. Thus, HU-induced reductions in splenocytes and thymocytes are each regulated by different mechanisms.

\section{Blocking the Fas-FasL interaction in HU-induced lymphocyte reduction}

We previously showed that blockade of the interaction of Fas and its ligand inhibits lymphocyte losses
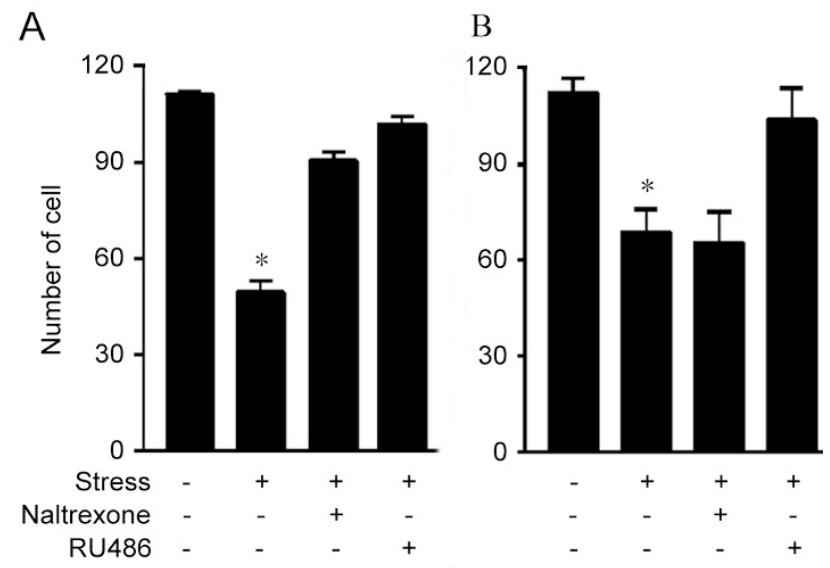

Fig 2. Effect of blocking corticosteroid receptors and opioid receptors on HU-induced lymphocyte reduction. Mice were treated with naltrexone (an opioid receptor blocker) or RU486 (a steroid receptor antagonist) daily while subjected to HU for 2 days, and the effect on splenic (A) and thymic (B) cellularity measured by counting total

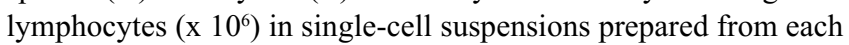
organ. HU-induced splenocyte reductions were reversed by either naltrexone or RU486, while thymocytes were rescued only by RU486. ${ }^{*} \mathrm{p}<0.01$ for HU-only group vs. control, $\mathrm{HU}+$ naltrexone, and $\mathrm{HU}+$ RU486 in spleen (A), and for HU-only group vs. control and HU + RU486 in thymus (B). 
due to restraint stress, a result confirmed by a recent study[44]. To investigate the effect of increased Fas activity on lymphocyte depletion in HU, FasL was neutralized by administering a FasL-specific antibody or FasFc fusion protein, a soluble form of Fas which binds FasL, before a 2-day HU-treatment. Both agents prevented HU-induced splenocyte reduction, but had no effect on thymocyte reduction (Fig 3 and data not shown) indicating that splenocyte loss from HU treatment is mediated by the Fas-FasL interaction, while thymocyte loss is not. After a full week of hindlimb suspension, however, this intervention became less effective with splenocytes (data not shown), suggesting that besides the Fas-FasL interaction, there may be additional mechanism(s), such as free radicals, that also control lymphocyte survival.

A
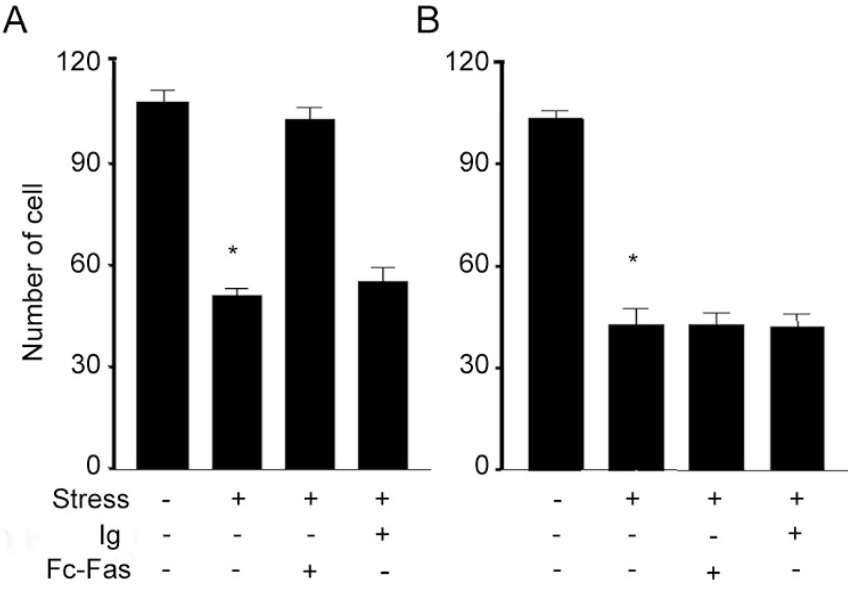

Fig 3. Effect of blocking the Fas-FasL interaction on HU-induced lymphocyte reduction. Male Balb/c mice were subjected to HU for $2 \mathrm{~d}$. with or without administration of Fas-Fc or control Ig (150 $\mu \mathrm{g}$

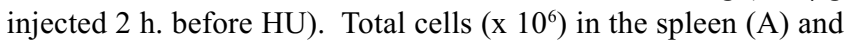
thymus (B) were enumerated. Fas-Fc treatment prevented HUinduced drop in splenocyte number, but had no effect on thymocyte reduction. $* \mathrm{p}<0.01$ for $\mathrm{HU}$-only vs. control and $\mathrm{HU}+\mathrm{Fas}-\mathrm{Fc}$ in spleen (A), and HU-only vs. control in thymus (B).

\section{DISCUSSION}

The immune system is affected by stress via hormonal and neuronal pathways in response to various physiological stressors including space flight. A number of laboratories have demonstrated that space flight has profound effects on immunological parameters in humans and animals, such as leukocyte blastogenesis, natural killer cell activity, leukocyte subset distribution, cytokine production, macrophage maturation and activity, monocytosis and lymphopenia. Due to severely limited access to outer space for experimental purposes, however, the molecular mechanisms underlying these changes remain unknown. Hindlimb unloading (HU) of rodents is a well-accepted ground-based model system that simulates some of the deleterious conditions of space flight. Here, we used this model to show that splenocyte and thymocyte numbers are dramatically reduced in HU-treated mice. The lymphocyte reductions in these organs are in part a consequence of increased apoptosis. Blocking of opioid receptors or the Fas-FasL interaction nearly eliminated splenocyte reduction, while thymocyte reduction was unchanged. But pretreatment with RU486, a steroid receptor antagonist, prevented depletion of both splenocytes and thymocytes. Thus, our results indicate that reductions in both splenocyte and thymocyte numbers that result from a chronic stress like HU are dependent on glucocorticoids, but only splenocyte depletion occurs mainly through endogenous opioid-dependent Fas-mediated apoptosis.

The immune system is compartmentalized into different organs and the stress response is unique in each. We observed that hormones of the hypothalamic-pituitaryadrenal (HPA) axis and endogenous opioids have divergent roles in the spleen and thymus of HU-treated mice. In this model, lymphocyte reduction in spleen is likely due to Fas-mediated apoptosis, but not so in the thymus. We also found that endogenous opioids affect these organs differently.

The interactions of the neuroendocrine system with the immune system and its importance in mediating disease are receiving increasing attention[10]. The primary hormonal pathway by which the central nervous system regulates the function of the immune system is the HPA axis[1]. Glucocorticoids are the final effectors of the HPA axis and participate in the control of whole body homeostasis as well as the organisms' response to stress[1]. Glucocorticoids act through ubiquitous cytoplasmic receptors; upon binding their ligand, glucocorticoid receptors translocate to the nucleus, where they interact as homodimers with specific glucocorticoid responsive elements within DNA to activate appropriate hormone-responsive genes. We observed that pretreatment with RU486, a steroid receptor antagonist, prevented depletion of both splenocytes and thymocytes in response to HU treatment.

Stress induces the production of various hormones and neuropeptides[44]. Glucocorticoids and endogenous opioids are the best-recognized of these mediators that 
modulate the immune response. Glucocorticoids play a critical role in modulating various physiological processes under stress conditions. Endogenous opioids are known to be elevated by stress and play a critical role in regulating stress-induced changes in the immune system. It is known that opioids induce the production of glucocorticoids via the HPA axis. Recent study has revealed that stress-induced lymphocyte reduction in spleen is dependent on endogenous opioids[44]. The interaction of glucocorticoids and endogenous opioids in stress-induced effects on immune system remain to be elucidated.

\section{ACKNOWLEDGEMENTS}

We thank Dr. Gerald Sonnenfeld for his advice on the establishment of the hindlimb-unloading model, and Drs. William Shearer and Sidney Pestka for their intellectual discussions. Drs. Satish Devadas, Zengrong Yuan and Ms. Cathy Liu provided invaluable technical assistance. Ms. Julie Amenta prepared histology sections. This project was supported by NIH grants AI43384 and AI50222, and the National Space Biomedical Research Institute of USA (IIH00208).

\section{REFERENCES}

1 Webster JI, Tonelli L, Sternberg EM. Neuroendocrine regulation of immunity. Annu Rev Immunol 2002; 20:125-63.

2 Harbuz M. Neuroendocrine-immune interactions. Trends Endocrinol Metab 2003; 14:51-2.

3 Gonzalez-Quijano MI, Martin M, Millan S Lopez-Calderon A. Lymphocyte response to mitogens: influence of life events and personality. Neuropsychobiology 1998; 38:90-6.

4 Husband AJ. Role of central nervous system and behaviour in the immune response. Vaccine 1993; 11:805-16.

5 Pedersen BK, Hoffman-Goetz L. Exercise and the immune system: regulation, integration, and adaptation. Physiol Rev 2000; 80:1055-81.

6 Spangelo BL, Judd AM, Call GB, Zumwalt J, Gorospe WC. Role of the cytokines in the hypothalamic-pituitary-adrenal and gonadal axes. Neuroimmunomodulation 1995; 2:299-312.

7 Maier, SF. Bi-directional immune-brain communication: Implications for understanding stress, pain, and cognition. Brain Behav Immun 2003; 17:69-85.

8 Marz P, Cheng JG, Gadient RA, et al. Sympathetic neurons can produce and respond to interleukin 6. Proc Natl Acad Sci USA 1998; 95:3251-6.

9 Shan J, Stachniak T, Jhamandas JH, Krukoff TL. Autonomic and neuroendocrine actions of adrenomedullin in the brain: mechanisms for homeostasis. Regul Pept 2003; 112:33-40.

10 Tsigos C, Chrousos GP. Hypothalamic-pituitary-adrenal axis, neuroendocrine factors and stress. J Psychosom Res 2002; 53: 865-71.

11 Haddad JJ, Saade NE, Safieh-Garabedian B. Cytokines and neuro-immune-endocrine interactions: a role for the hypothalamic-pituitary-adrenal revolving axis. J Neuroimmunol 2002; 133:1-19.

12 Ader R, Cohen N, Felten D. Psychoneuroimmunology: interactions between the nervous system and the immune system. Lancet 1995; 345:99-103.

13 Ben-Eliyahu $\mathrm{S}$. The promotion of tumor metastasis by surgery and stress: immunological basis and implications for psychoneuroimmunology. Brain Behav Immun 2003; 17 Suppl 1: S27-36.

14 Stefanski V. Social stress in laboratory rats: behavior, immune function, and tumor metastasis. Physiol Behav 2001. 73:38591.

15 Sheridan JF, Dobbs C, Jung J, Chu X, Konstantinos A, Padgett D, Glaser R. Stress-induced neuroendocrine modulation of viral pathogenesis and immunity. Ann N Y Acad Sci 1998; 840: 803-8.

16 Shanks N, Kusnecov AW. Differential immune reactivity to stress in BALB/cByJ and C57BL/6J mice: in vivo dependence on macrophages. Physiol Behav 1998; 65:95-103.

17 Vaccarino AL, Olson GA, Olson RD, Kastin AJ. Endogenous opiates: 1998. Peptides 1999; 20:1527-4.

18 Nieman DC. Exercise immunology: practical applications. Int J Sports Med 1997; 18 Suppl 1:S91-100.

19 Nieman DC, Pedersen BK. Exercise and immune function. Recent developments. Sports Med 1999; 27:73-80.

20 Pariante CM, Carpiniello B, Orru MG, et al. Chronic caregiving stress alters peripheral blood immune parameters: the role of age and severity of stress. Psychother Psychosom 1997; 66: 199-207.

21 Berthiaume F, Aparicio CL, Eungdamrong J, Yarmush ML. Age- and disease-related decline in immune function: an opportunity for "thymus-boosting" therapies. Tissue Eng 1999; 5: 499-514.

22 Zorrilla EP, Valdez GR, Weiss F. Changes in levels of regional CRF-like-immunoreactivity and plasma corticosterone during protracted drug withdrawal in dependent rats. Psychopharmacology (Berl) 2001; 158:374-81.

23 Iwagaki H, Morimoto Y, Kodera M, Tanaka N. [Surgical stress and CARS: involvement of T cell loss due to apoptosis]. Rinsho Byori 2000; 48:505-9.

24 Pedersen BK, Bruunsgaard H, Jensen M, Toft AD, Hansen H, Ostrowski K. Exercise and the immune system-influence of nutrition and ageing. J Sci Med Sport 1999; 2:234-52.

25 Zakowski SG, McAllister CG, Deal M, Baum A. Stress, reactivity, and immune function in healthy men. Health Psychol 1992; 11:223-32.

26 Capitanio AM, Sacco R, Mannucci PM. Pseudopathologies of hemostasis and dental surgery. Oral Surg Oral Med Oral Pathol 1991; 71:184-6.

27 Padgett DA, Sheridan JF, Dorne J, Berntson GG, Candelora J, Glaser R. Social stress and the reactivation of latent herpes simplex virus type 1. Proc Natl Acad Sci USA 1998; 95:7231-5.

28 Wyllie AH, Kerr JF, Currie AR. Cell death: the significance of apoptosis. Int Rev Cytol 1980; 68:251-306.

29 Sun EW, Shi YF. Apoptosis: the quiet death silences the immune system. Pharmacol Ther 2001; 92:135-45.

30 Shi YF, Devadas S, Greeneltch KM, Yin D, Allan Mufson R, Zhou JN. Stressed to death: implication of lymphocyte 
apoptosis for psychoneuroimmunology. Brain Behav Immun 2003; 17 Suppl 1:S18-26.

31 Rich T, Watson CJ, Wyllie, A. Apoptosis: the germs of death. Nat Cell Biol 1999; 1:E69-71.

32 Green DR, Ferguson, TA. The role of Fas ligand in immune privilege. Nat Rev Mol Cell Biol 2001; 2:917-24.

33 Shi YF, Sahai BM, Green DR. Cyclosporin A inhibits activation-induced cell death in T-cell hybridomas and thymocytes. Nature 1989; 339:625-6.

34 Shi YF, Bissonnette RP, Parfrey N, Szalay M, Kubo RT, Green DR. In vivo administration of monoclonal antibodies to the CD3 T cell receptor complex induces cell death (apoptosis) in immature thymocytes. J Immunol 1991; 146:3340-6.

35 Smith CA, Williams GT, Kingston R, Jenkinson EJ, Owen JJ. Antibodies to CD3/T-cell receptor complex induce death by apoptosis in immature T cells in thymic cultures. Nature 1989; 337:181-4.

36 Lin YS, Lei HY, Low TL, Shen CL, Chou LJ, Jan MS. In vivo induction of apoptosis in immature thymocytes by staphylococcal enterotoxin B. J Immunol 1992; 149:1156-63.

37 Lenardo, MJ. Interleukin-2 programs mouse alpha beta T lymphocytes for apoptosis. Nature 1991; 353:858-61.

38 Wesselborg S, Kabelitz D. Activation-driven death of human T cell clones: time course kinetics of the induction of cell shrinkage, DNA fragmentation, and cell death. Cell Immunol 1993; 148: 234-41.

39 Nagata, S. Fas and fas ligand: a death factor and its receptor. Adv Immunol 1994; 57:129-44

40 Takahashi T, Tanaka M, Brannan CI, et al. Generalized lymphoproliferative disease in mice, caused by a point mutation in the Fas ligand. Cell 1994; 76:969-76.

41 Takahashi T, Tanaka M, Inazawa J, Abe T, Suda T, Nagata S. Human Fas ligand: gene structure, chromosomal location and species specificity. Int Immunol 1994; 6:1567-74.

42 Lenardo MJ. Molecular regulation of T lymphocyte homeostasis in the health and diseased immune system. Immunol Res 2003; 27:387-98

43 Yin D, Mufson RA, Wang R, Shi Y. Fas-mediated cell death promoted by opioids. Nature 1999; 397:218.

44 Yin D, Tuthill D, Mufson RA, Shi Y. Chronic restraint stress promotes lymphocyte apoptosis by modulating CD95 expression. J Exp Med 2000; 191:1423-8.

45 Sonnenfeld G, Butel JS, Shearer WT. Effects of space flight environment on the immune system. Rev Environ Health 2003; 18:1-17.

46 Chapes SK, Mastro AM, Sonnenfeld G, Berry WD. Antiorthostatic suspension as a model for the effects of spaceflight on the immune system. J Leukoc Biol 1993; 54:227-35.

47 Belay T, Aviles H, Vance M, Fountain K, Sonnenfeld G. Effects of the hindlimb-unloading model of spaceflight conditions on resistance of mice to infection with Klebsiella pneumoniae. J Allergy Clin Immunol 2002; 110:262-8.

48 Zhang H, Yang Y, Horton JL, et al. Amelioration of collageninduced arthritis by CD95 (Apo-1/Fas)-ligand gene transfer. J Clin Invest 1997; 100:1951-7.

49 Wang, J, Charboneau R, Barke RA, Loh HH, Roy S. Muopioid receptor mediates chronic restraint stress-induced lymphocyte apoptosis. J Immunol 2002; 169:3630-6.

50 Pierzchala-Koziec K, Niezgoda J, Bobek S, Przewlocki R. Endogenous opioids may modulate the activity of the hypothalamus- pituitary-adrenocortical axis in domestic fowl. Zentralbl Veterinarmed A 1996; 43:139-46.

51 Mosconi G, Carnevali O, Facchinetti, F, Neri I, PolzonettiMagni A. Opioid peptide modulation of stress-induced plasma steroid changes in the frog Rana esculenta. Horm Behav 1994; 28:130-8 